\title{
Multispectral X-ray CT: multivariate statistical analysis for efficient reconstruction
}

\author{
Kheirabadi, Mina; Mustafa, Wail; Lyksborg, Mark; Olsen, Ulrik Lund; Dahl, Anders Bjorholm
}

Published in:

Proceedings Volume 10391, Developments in X-Ray Tomography XI

Link to article, DOI:

$10.1117 / 12.2273338$

Publication date:

2017

Document Version

Publisher's PDF, also known as Version of record

Link back to DTU Orbit

Citation (APA):

Kheirabadi, M., Mustafa, W., Lyksborg, M., Olsen, U. L., \& Dahl, A. B. (2017). Multispectral x-ray CT: multivariate statistical analysis for efficient reconstruction. In Proceedings Volume 10391, Developments in XRay Tomography XI SPIE - International Society for Optical Engineering. Proceedings of SPIE - The International Society for Optical Engineering https://doi.org/10.1117/12.2273338

\section{General rights}

Copyright and moral rights for the publications made accessible in the public portal are retained by the authors and/or other copyright owners and it is a condition of accessing publications that users recognise and abide by the legal requirements associated with these rights.

- Users may download and print one copy of any publication from the public portal for the purpose of private study or research.

- You may not further distribute the material or use it for any profit-making activity or commercial gain

- You may freely distribute the URL identifying the publication in the public portal 


\section{Multispectral x-ray CT: multivariate statistical analysis for efficient reconstruction}

Mina Kheirabadi

Wail Mustafa

Mark Lyksborg

Ulrik Lund Olsen

Anders Bjorholm Dahl 


\title{
Multispectral X-ray CT - Multivariate Statistical Analysis for Efficient Reconstruction
}

\author{
Mina Kheirabadi ${ }^{\mathrm{a}}$, Wail Mustafa ${ }^{\mathrm{a}}$, Mark Lyksborga ${ }^{\mathrm{a}}$, Ulrik Lund Olsen ${ }^{\mathrm{b}}$, and Anders Bjorholm \\ Dahl $^{\mathrm{a}}$ \\ aDepartment of Applied Mathematics and Computer Science, Technical University of Denmark \\ ${ }^{\mathrm{b}}$ Department of Physics, Technical University of Denmark
}

\begin{abstract}
Recent developments in multispectral X-ray detectors allow for an efficient identification of materials based on their chemical composition. This has a range of applications including security inspection, which is our motivation. In this paper, we analyze data from a tomographic setup employing the MultiX detector, that records projection data in 128 energy bins covering the range from 20 to $160 \mathrm{keV}$. Obtaining all information from this data requires reconstructing 128 tomograms, which is computationally expensive. Instead, we propose to reduce the dimensionality of projection data prior to reconstruction and reconstruct from the reduced data. We analyze three linear methods for dimensionality reduction using a dataset with 37 equally-spaced projection angles. Four bottles with different materials are recorded for which we are able to obtain similar discrimination of their content using a very reduced subset of tomograms compared to the 128 tomograms that would otherwise be needed without dimensionality reduction.
\end{abstract}

Keywords: Multispectral X-ray, Tomography, Multivariate statistical analysis, Dimensionality reduction

\section{INTRODUCTION}

Multispectral X-ray detectors allow for the detection of materials with higher chemical specificity than methods based on single or dual energy, ${ }^{1}$ which is the current standard. Multispectral X-ray imaging has a range of applications in e.g. food inspection ${ }^{2}$ or security, ${ }^{3}$ where the latter motivates our work. Most security applications are based on 2D radiography, but some applications such as check-in baggage inspection at airports employ 3D scanning. ${ }^{4}$ 3D scanning provides detailed images of the internal structures of the baggage. This solves some of the problems in 2D scanning caused by, for instance, occlusion. 3D scanning requires tomographic reconstruction, ${ }^{5}$ which is computationally expensive and therefore time-consuming. This is a major problem for rapid baggage inspection.

Baggage inspection is an important part of airport security, and detecting prohibited items and especially explosives is a major concern. Nowadays, baggage inspection is mostly done manually by looking at the acquired images, but there is an increasing interest in automated methods for detecting threat items. ${ }^{4}$ Until now, there has been limited academic interest in developing automated methods for inspection, which might be due to limited access to data ${ }^{6}$ and a lack of regulatory requirements. Data is essential for developing methods for detection of objects like handguns, knives, etc. based on their appearance. Appearance-based recognition methods use e.g. feature-based classification ${ }^{7}$ or deep learning ${ }^{8}$ from single or dual energy images. ${ }^{9,10}$ For explosive detection based on this type of object recognition methods, a very large set of training data is required containing images of explosives, detonators, and wires. Even with the availability of such data, the problem will still be very complicated, despite the recent advances in object recognition, because many legal items have shapes that are very close to explosives. This will however be a much easier recognition task if the threat items can be distinguished by their unique patterns of X-ray spectral absorption. ${ }^{11,12}$

Constructing a scanner with fixed detectors has the advantage of being mechanically stable, and can support a longer exposure time for the detector and thus a higher signal to noise ratio in the projections as opposed

Further author information: (Send correspondence to M. Kheirabadi or W. Mustafa)

M. Kheirabadi: E-mail: mink@dtu.dk, W. Mustafa: E-mail: wamus@dtu.dk, Telephone: +45 45253407

Developments in X-Ray Tomography XI, edited by Bert Müller, Ge Wang, Proc. of SPIE Vol. 10391, 1039113 - (c) 2017 SPIE · CCC code: 0277-786X/17/\$18 · doi: 10.1117/12.2273338 
to a system with a rotating gantry. A fixed gantry setup however typically requires individual detectors and sources for each projection. This means installing several multispectral detectors, which are relatively expensive compared to traditional single or dual energy detectors. Therefore, there is an incentive to use the least number of detectors possible, resulting in few projections available for the tomographic reconstruction. Reconstruction from few projections using a standard back-projection type of algorithm often results in poor image quality. In such situations, using regularized algebraic techniques often leads to significantly improved quality. ${ }^{13}$ Since the required reconstruction is time costly, it is a drawback if all individual energy channels from the multispectral sensor should be reconstructed individually. To overcome this, we propose to limit the number of tomograms needed for obtaining the material specific information. Here, we investigate how this can be achieved by reducing the dimensionality of the projection data and thereby avoiding to reconstruct all energy channels. This dimensionality reduction is done using multivariate statistical analysis methods including principal component analysis and non-negative matrix factorization. For comparison, we also bin the energy channels.

Other works have been carried out for reducing the complexity of multispectral X-ray CT. Eger et al. ${ }^{14}$ studied the problem of classifying materials as explosives or not using spectra of linear attenuation coefficients from 10 to $150 \mathrm{keV}$. They showed that explosives can be detected using sequential linear discriminant analysis of data containing three features. This study, however, does not consider the noise and artifacts introduced by computed tomography. In Lee et al. ${ }^{15}$ they performed a study based on a CdTe detector using six spectral bands in the range from 22 to $44 \mathrm{keV}$. Their experiment was done using a physical phantom made of a cylinder with four holes containing different materials, and the cylinder itself as the fifth material. In this respect, their study is similar to ours, but they employed 320 projections, as opposed to our 37 . However, our MultiX detector, ${ }^{3,16}$ which is also a CdTe detector, returns 128 spectral bands, so exploiting the information from this data directly requires reconstructing 128 tomograms. Instead, we investigate if we can retain the information by reducing the dimensionality of the projection data prior to reconstruction.

Our analysis is based on data from our experimental X-ray setup employing a MultiX CdTe detector providing 128 energy channels in the range from 20 to $160 \mathrm{keV}$. Our physical phantom contains four glass bottles with different materials, and we investigate if we can obtain a signal that allows us to discriminate the materials. Tomographic reconstruction is the computationally most expensive part, so we investigate if we can get a sufficiently discriminative signal by only reconstructing a few tomograms instead of all 128 . This is done by reducing the dimensionality of the projection data.

\section{DIMENSIONALITY REDUCTION METHODS}

The direct approach for classifying materials from multispectral X-ray tomography is to reconstruct all energy channels individually and carry out the classification on this data (Figure 1a), which we use as our reference. To obtain this, we must choose a reconstruction method and a classification technique. These methods, however, are not the focus of our investigation. In this work, we have chosen to employ an iterative algebraic reconstruction technique with a total variation regularization (ART-TV) ${ }^{17}$ and performing a pixel-wise classification using linear discriminant analysis. The typical choice for reconstruction would be a back-projection algorithm, e.g., filtered back-projection (FBP), but the limited number of projections did not give sufficient quality using this type of method, and we obtained satisfactory results using ART-TV. We realize that the choice of reconstruction method will influence the obtained result, but we will leave that for later studies. Also for classification, it might be better choosing a more sophisticated classification method, but because we only investigate one dataset, we have only few pixels from the same image to test the method. Therefore, the classification method is used primarily to assess if we obtain a separation of data points from the different materials. In a real setting, a number of factors would influence the obtained images, e.g. variation in content, variation in system sensitivity over time, etc., which are not investigated here.

Reducing the 128-dimensional projection data can be done in a number of ways. A simple approach is to bin the data which simulates a detector with fewer spectral bins. A drawback is that not all spectral information is utilized. Therefore, we also investigate other linear methods for dimensionality reduction that aim to find latent representations expressing most of the information but with fewer dimensions. In this section, we describe those methods as applied for reducing the dimensionality of the projection data (Figure 1b), since the goal of this 

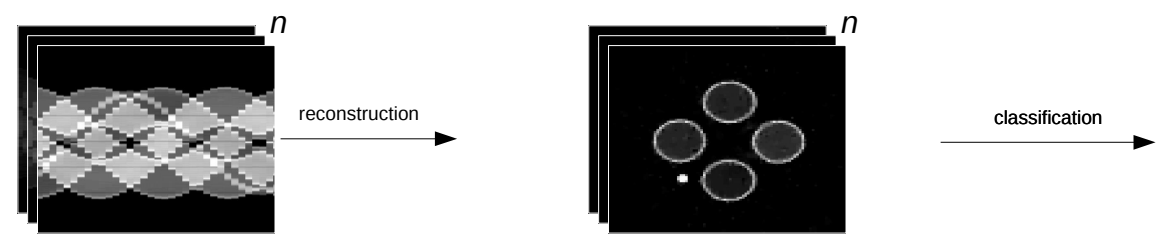

(a) No reduction

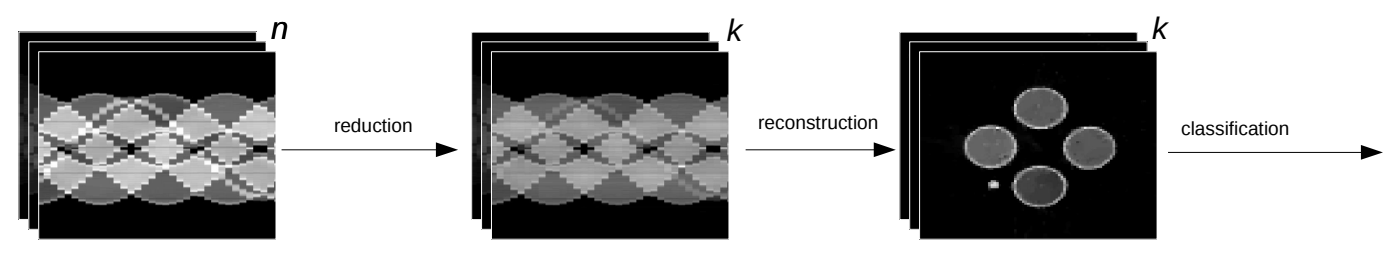

(b) Pre-reconstruction reduction

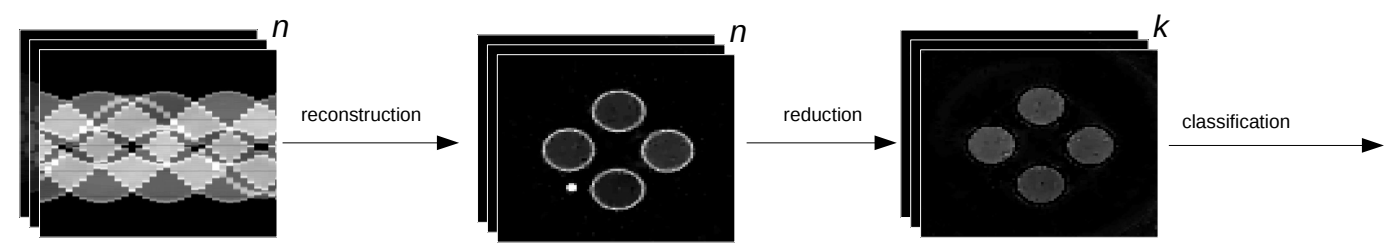

(c) Post-reconstruction reduction

Figure 1: Tomographic reconstruction and classification from multispectral projection data of $n$ channels. (a) No reduction is performed, requiring to reconstruct all channels. (b) Projection data is reduced to $k$ channels prior to reconstruction. (c) Reduction is performed on the reconstructed images.

paper is to investigate the application of this approach to reduce the required number of reconstructions. In our experiments and as a reference, we also applied those methods on the reconstructed images (Figure 1c).

With dimensionality reduction, we transform a matrix $\boldsymbol{B} \in R^{m \times n}$, where $m$ corresponds to measurements (or observations) and $n$ corresponds to dimensions (or variables), to $\boldsymbol{B}_{\text {reduced }} \in R^{m \times k}$ where $k$ corresponds to the number of dimensions after reduction. For our projection data, $n=128$, which is the number of multispectral bins and $m$ is the total number of projection data points measured by the detector pixels. In our experiment, we use two Multix detectors with 128 pixels each to record data from 37 projection angles, and hence $m=9472$.

\subsection{Data binning}

Data binning is simply done by averaging the 128 projections in $n$ spectral bins. It is easy to vary the number of bins and measure the effect, but choosing the optimal number of bins and their specific combinations is a combinatorial problem and therefore challenging. Instead, we have tried different other techniques that maps the data to a subspace according to different optimality criteria.

\subsection{Non-negative matrix factorization}

Non-negativity matrix factorization (NMF) is a well-known tool in multivariate statistics to decompose inherently non-negative data into basis factors. ${ }^{18}$ The method factorizes a non-negative matrix, here $\boldsymbol{B} \in R^{m \times n}$, into two non-negative matrices $\boldsymbol{W} \in R^{m \times k}$ and $\boldsymbol{H} \in R^{k \times n}$ such that $\boldsymbol{B} \approx \boldsymbol{W} \boldsymbol{H}$ where $k$ is the number of factors, which generally must be chosen to be smaller than $m$ or $n$. By doing this factorization, the $n$ columns of the original matrix $\boldsymbol{B}$ become approximated with a linear combination of the $k$ columns of $\boldsymbol{W}$ weighted by the $n$ columns of $\boldsymbol{H}$. This means that the $(n-k)$ fewer columns of $\boldsymbol{W}$ can be used to express the original $n$ columns. In other words, $\boldsymbol{W}$ can be regarded as a reduced representation of our multispectral projection data. For this to be a good approximation, we aim to find factors that correspond to latent structures in the data, which translate in our case to discriminative information. 
Because there is no exact solution to this factorization problem, existing algorithms seek an approximation that minimizes a certain cost function. In this paper, we use the standard algorithm provided by MATLAB. The algorithm ${ }^{18}$ minimizes the root-mean-squared residual $\boldsymbol{D}$ of $\boldsymbol{B}$ and $\boldsymbol{W} \boldsymbol{H}$ where:

$$
\boldsymbol{D}=\frac{\|\boldsymbol{B}-\boldsymbol{W} \boldsymbol{H}\|_{2}}{\sqrt{n m}} .
$$

The algorithm employs the alternating least squares (ALS) method to solve this optimization problem iteratively. The method starts by generating random initial values for $\boldsymbol{W}$ and $\boldsymbol{H}$. At each iteration, the least squares solution of one matrix is computed, given the other matrix. Also, the non-negativity is enforced by setting all negative elements to 0 .

\subsection{Principle component analysis}

Principle component analysis $(\mathrm{PCA})^{19}$ is arguably the most popular method for multivariate dimensionality reduction. The objective of PCA is to find a linear transformation that maps data expressed with potentially correlated variables into a new set expressed by linearly uncorrelated variables, called principal components. The transformation is sought to maximize the total variance and constrained by the orthogonality of the principle components. The principal components are ordered according to their associated variance and only the first $k$ components are kept when performing dimensionality reduction. In other words, PCA allows for capturing the maximum variability in the data and that is often linked to preserving desired features (or compressed information). In our context, PCA transforms the projection data, expressed by $n$ correlated energy bins into a reduced subspace of $k$ bins.

Given the projection data $\boldsymbol{B} \in R^{m \times n}$, PCA finds the matrix $\boldsymbol{P} \in R^{n \times n}$ that transforms $\boldsymbol{B}$ to $\boldsymbol{Y} \in R^{m \times n}$. The matrix $\boldsymbol{P}$, also called the loadings, describes the relations between the variables (here, the original energy bins) and the components. The direct approach for finding this transformation is to first compute the $n$ eigenvectors and eigenvalues of the variance-covariance (dispersion) matrix of $\boldsymbol{B}, \boldsymbol{D}(\boldsymbol{B}) \in R^{n \times n}$. Then, the eigenvectors are sorted according to their respective eigenvalues in a descending order. The matrix $\boldsymbol{P}$ is finally composed of the sorted eigenvectors. To reduce the dimensionality, the first $k$ eigenvectors are kept, reducing the $\boldsymbol{P}$ to $\boldsymbol{P}_{\boldsymbol{r}} \in R^{n \times k}$. In this paper, we use the standard PCA provided by MATLAB in which the eigenvalues and eigenvectors are computed using the singular value decomposition (SVD) algorithm.

\subsubsection{PCA with varimax rotation}

Sometimes, revealing the latent structures in the data may be best achieved by redistributing the variance over the $k$ components. ${ }^{19}$ This can be done by simply rotating $\boldsymbol{P}_{\boldsymbol{r}}$ in its subspace. This means that we convert the complex relationship between the components and the energy bins where each component tends to be associated with a large number of energy bins, to a simpler one where each component may be associated with a smaller number of energy bins. For finding this optimal rotation, we apply the varimax method, ${ }^{20}$ which maximizes the sum of the variances of the squared columns of $\boldsymbol{P}_{\boldsymbol{r}}$ while preserving orthogonality.

\section{EXPERIMENTS}

The goal of the experiments we conduct in this paper is to study if we can retain discriminative information after reducing the dimensionality of the projection data prior to reconstruction. We study that on a physical phantom containing four different materials. The data is acquired using an experimental multispectral X-ray setup.

\subsection{Multispectral X-ray setup}

All X-ray data are acquired at a general-purpose spectral X-ray acquisition setup built at The Technical University of Denmark (DTU). ${ }^{21}$ In the order of beam propagation, the setup consists of an X-ray generator; a primary slit; a sample stage featuring $x, y, z$ and rotation movement; a secondary detector slit and a $1 \mathrm{D}$ detector. The generator is a Comet MXR- $160 \mathrm{HP} / 11$ operated at $160 \mathrm{kVp}$ and $0.5 \mathrm{~mA}$. The primary slit, positioned $246 \mathrm{~mm}$ from the focal spot, is a JJ X-ray IB-30-Air aligned for a fan beam with $0.6 \mathrm{~mm}$ beam height and $30 \mathrm{~mm}$ beam 

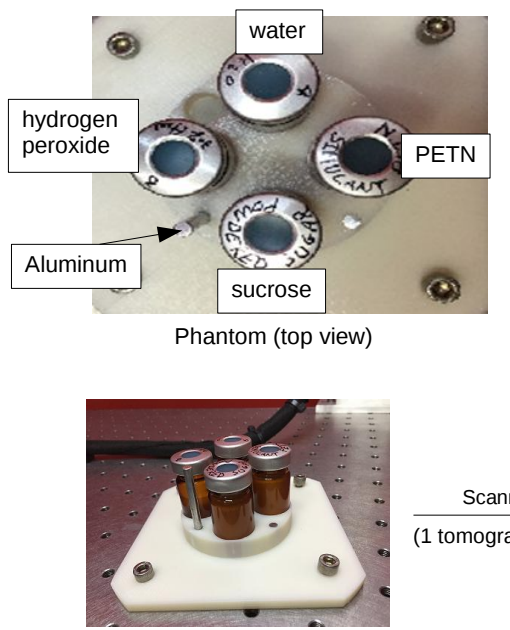

Dataset phantom
Projection sinograms
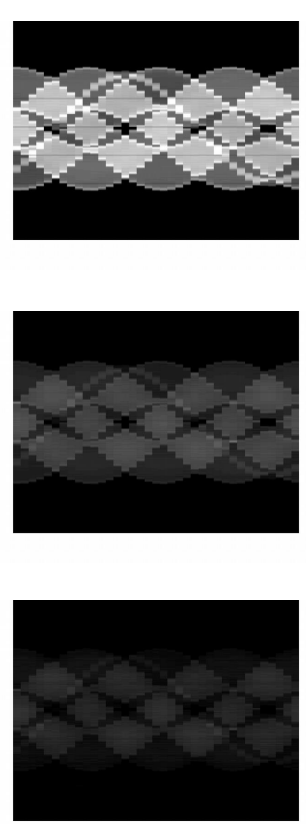

Reconstructed images

$(30 \mathrm{keV})$
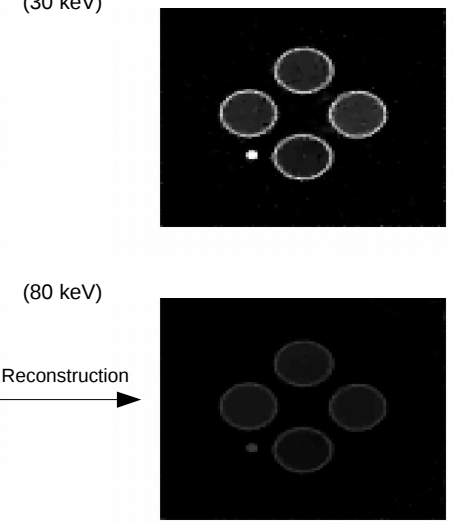

$(149 \mathrm{keV})$

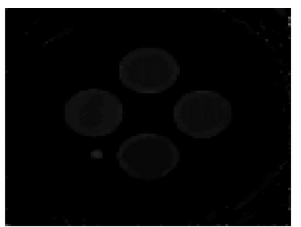

Figure 2: Phantom used in the experiments (left). Only one tomographic slice is acquired. Projection data at three energy levels and the corresponding reconstructed images are shown as examples. The full set contains data from 128 bins covering the range from 20 to $160 \mathrm{keV}$. In the classification experiment, we consider four materials: water, hydrogen peroxide, sucrose and PETN. The four materials are segmented out manually

width. The sample is positioned $84 \mathrm{~cm}$ from the source and the detector is $82 \mathrm{~cm}$ farther from the sample. The secondary slit is custom Tungsten blades placed $10 \mathrm{~mm}$ in front of the detector window with an opening of $0.6 \mathrm{~mm}$, which is slightly less than the pixel size of $0.8 \mathrm{~mm}$. The detector consists of two tiled modules of Multix Me-100 V2 using an $3 \mathrm{~mm}$ thick CdTe detector crystal. Each module has 128 pixels and separates the photons in 128 energy bins in the range 20 to $160 \mathrm{keV}$. Energy resolution of the detector is $7 \mathrm{keV}$ FWHM evaluated at $70 \mathrm{keV}$.

To acquire one tomographic slice, the sample is rotated and imaged at 37 angular positions evenly spaced over $360^{\circ}$. At each position, the sample projection is acquired 25 consecutive times each with a $400 \mathrm{~ms}$ exposure time. For each projection, the median value of each bin in each pixel is computed from the set of 25 equivalent acquisition. The detector response is corrected using a method developed by Christensen et al ${ }^{22}$ for spectral CT applications. The flat field measurement is performed subsequently in the same setup but with the sample removed.

\subsection{Dataset}

For our experiments, we created a physical phantom (Figure 2) of four glass bottles containing four different materials to classify. The materials are water $\left(\mathrm{H}_{2} \mathrm{O}\right), 30 \%$ hydrogen peroxide $\left(\mathrm{H}_{2} \mathrm{O}_{2}\right)$, sucrose $\left(\mathrm{C}_{12} \mathrm{H}_{22} \mathrm{O}_{11}\right)$ and PETN $\left(\mathrm{C}_{5} \mathrm{H}_{8} \mathrm{~N}_{4} \mathrm{O}_{12}\right)^{*}$. The four materials considered for classification were chosen because of their closeness in terms of their X-ray absorption, which makes their classification a challenging task that requires utilizing the multispectral data. Addressing this kind of task makes it easier to assess the extent to which discriminative information is maintained after reducing the dimensionality. The task also resembles real scenarios in baggage scanning, motivating our work, in which benign materials, such as water, can be confused as unsafe materials,

\footnotetext{
${ }^{*}$ X-ray correct simulant from Renful Premier Technologies
} 


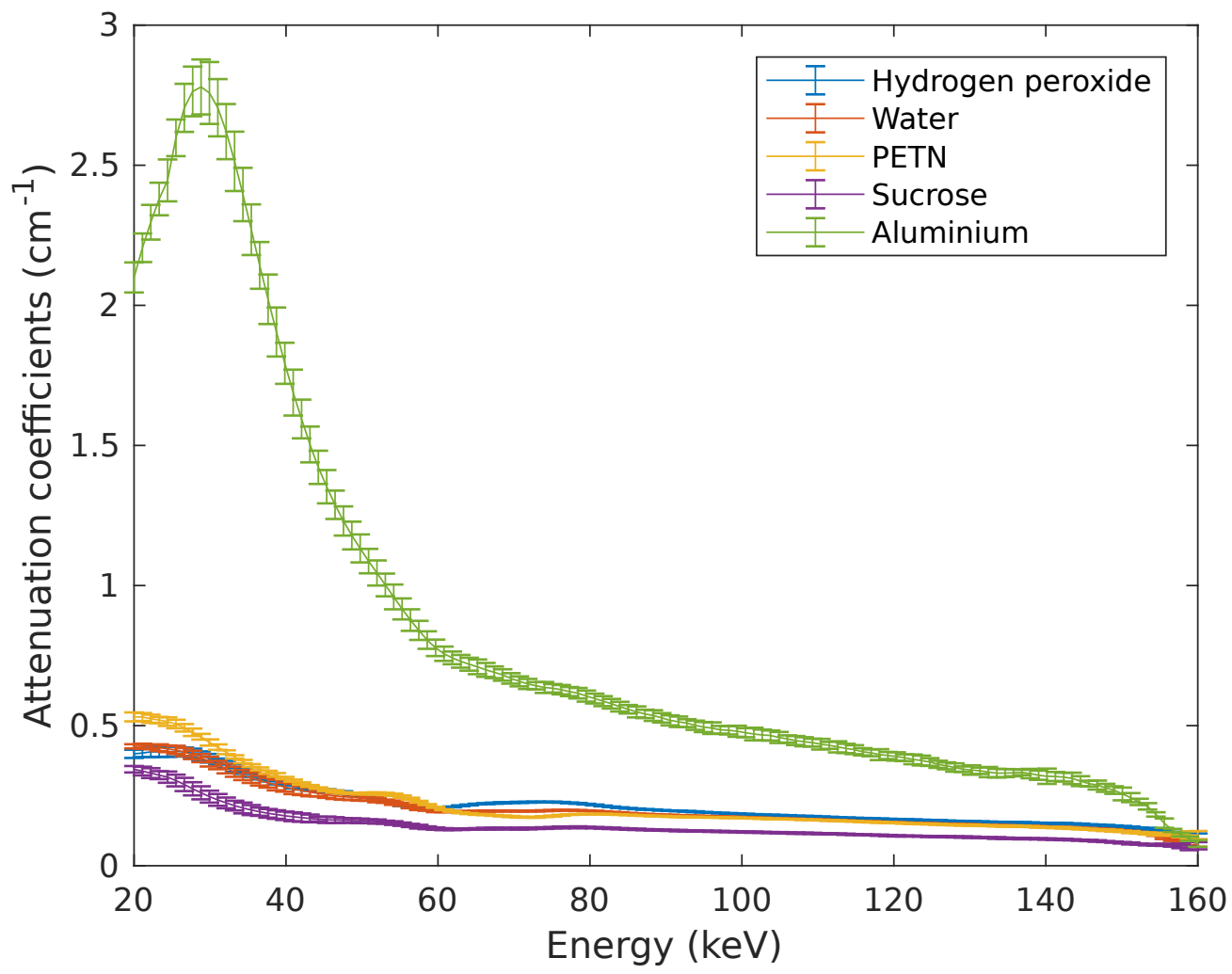

Figure 3: Attenuation coefficient curves of the materials in the dataset.

such as PETN. The phantom also includes an aluminum pin (Al), which we don't consider for classification since it is very different from the other materials.

Figure 3 depicts the attenuation coefficient curves of the four investigated materials and the aluminum (glass is not included). To obtain the curves, we reconstructed all energy bins of a tomographic slice taken from the phantom. Then, we computed the average intensity and the standard deviation of the pixels belonging to each material in the reconstructed images after manual segmentation. The figure shows that the attenuation coefficient values of water, hydrogen peroxide and PETN are particularly close.

\subsection{Classification experiment}

Pixel-wise classification of the four materials is used to assess if we can retain sufficient information to discriminate the materials after reducing the dimensionality of the projection data using the methods discussed in Sec. 2. To this end, we trained one classifier, namely, linear discriminate analysis (LDA). The feature vectors we feed into the classifier are constructed from the pixel intensity values across the energy bins. For simplicity, manual segmentation was carried out, resulting in 177 pixels for each material. At each classification run, 141 pixels are randomly selected out of 177 pixels for training while the remaining pixels are kept for testing.

As a performance metric, we calculate the misclassification rate of a material as the ratio of the wrongly classified test pixels. This routine is executed 20 times from which the average and the standard deviation of the misclassification rate are computed. To find the least number of dimensions needed for high and stable performance, we repeat the same experiment while we vary $k$, the number of dimensions we reduce the data to, from 1 to 10. 


\section{RESULTS AND DISCUSSION}

First in Figure 4, we show the classification performance when dimensionality reduction is performed on the projection data before reconstruction. Secondly in Figure 5 and as a matter of comparison, we show the classification performance when the reduction is performed on the reconstructed images after reconstruction. For the latter, the name of the reduction method is followed by 'post'. In both figures, we show the mean misclassification rate of the individual materials as well as the average of all materials, which we regard as the overall method performance for comparison purposes. The average method performance is summarized in Figure 6.

Figure 4 shows the misclassification rate when the number of dimensions is reduced. Reducing to 1 dimension has in all cases the highest misclassification rate. For 2 dimensions the misclassification rate drops significantly for all methods except for PCA and continue to monotonically decrease with higher dimensions. With 7 dimensions, both low misclassification rate and relatively low standard deviation are obtained. The results corresponding to this number of dimensions are compiled in Table 1.
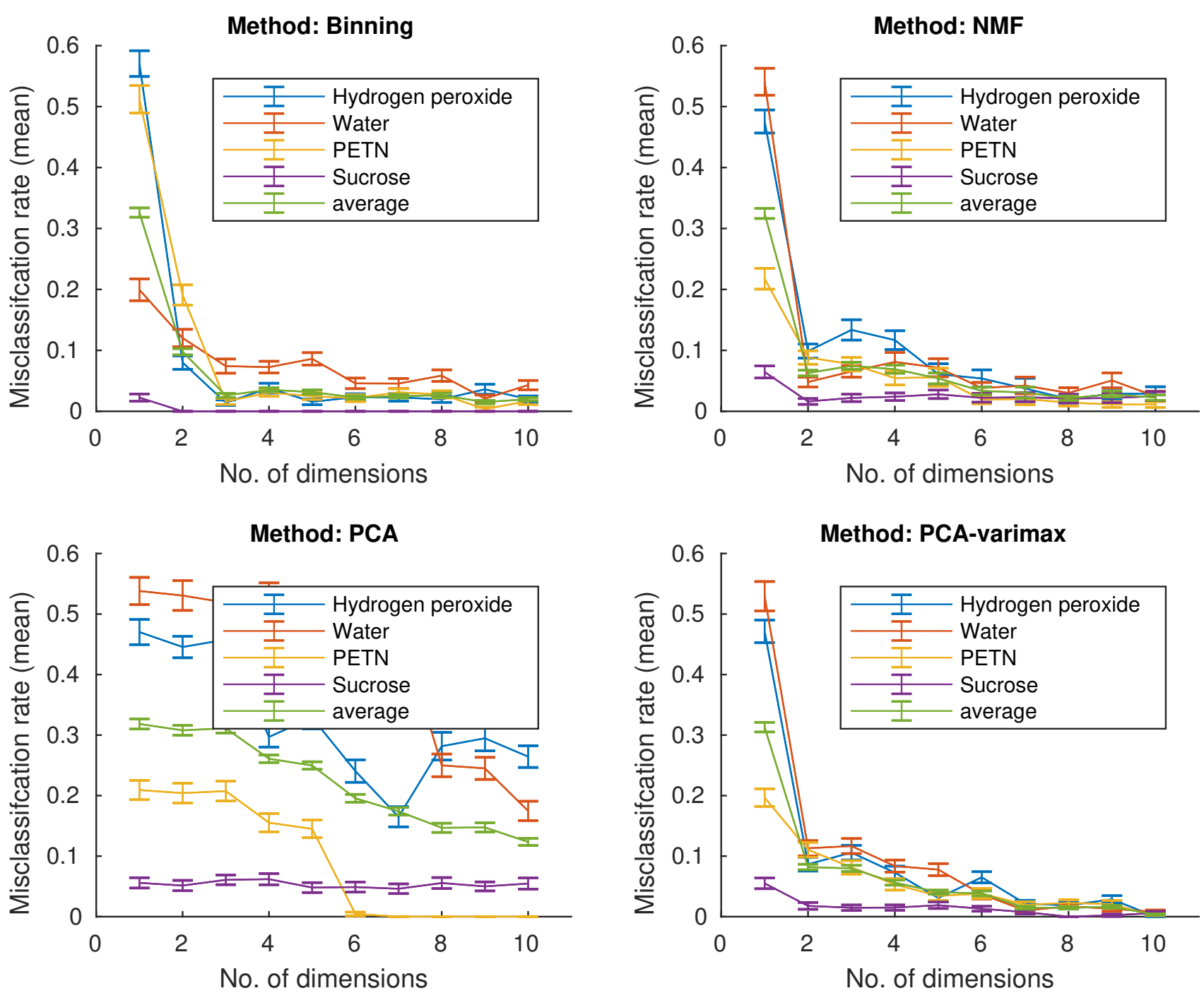

Figure 4: Classification performance when dimensionality reduction is performed on the projection data prior to image reconstruction (pre-reconstruction reduction). Bars represent the standard deviations, however for better visibility, one fourth of the actual standard deviation is shown. 


\begin{tabular}{|l|ccccr|}
\hline Method & Hydrogen Peroxide & Water & PETN & Sucrose & average \\
\hline \hline No reduction (128 bins) & 0 & 0 & 0 & 0 & 0 \\
\hline \hline Pre-reconstruction (7 bins) & & & & & \\
Binning & 0.02 & 0.05 & 0.03 & 0 & 0.03 \\
PCA & 0.17 & 0.49 & 0 & 0.046 & 0.18 \\
PCA-varimax & 0.02 & 0.01 & 0.02 & 0.01 & 0.02 \\
NMF & 0.04 & 0.04 & 0.02 & 0.02 & 0.03 \\
\hline \hline Post-reconstruction (7 bins) & & & & & \\
Binning & 0 & 0.01 & 0.01 & 0 & 0.01 \\
PCA & 0.01 & 0.02 & 0.01 & 0.02 & 0.02 \\
PCA-varimax & 0.01 & 0.02 & 0.0 & 0.01 & 0.02 \\
NMF & 0.01 & 0.03 & 0.01 & 0.01 & 0.02 \\
\hline \hline
\end{tabular}

Table 1: The average performance when dimensionality is reduced to 7 energy bins. The top row shows the performance when all energy bins are used.

While perfect classification is achieved when we reconstruct all the 128 energy bins, the results show that we obtain an average misclassification rate of only 0.02 when we reduce the projection data to 7 dimensions using PCA-varimax. This indicates that the multispectral information needed to distinguish the materials were largely retained despite the data being significantly reduced from 128 to 7 energy bins. This performance is also comparable to what we obtain if data reduction is performed on the reconstructed images. This suggests that the amount of information lost by data reduction is comparable in both cases and hence no undue information loss occurs when the reduction is performed prior to reconstruction.

When assessing the individual methods, we can observe that the different methods (except for PCA) achieve comparable performance. Conclusions on which method is the best may not be reached when the standard deviation is considered (note that only one fourth of the standard deviation is shown in the figures for better visibility). However considering the standard deviation in the relative sense, PCA-varimax shows to be more stable than NMF (best viewed in Figure 6). This may be because NMF generates random initial estimates (Subsec. 2.2), which might result in inconsistent factors at different runs. We can also observe that data binning results in comparable performance. Nevertheless, it is expected that for more complex data, simple binning will cause significant loss of information.

It is clear that PCA results in poor performance when used to reduce the dimensionality of the projection data. This indicates, at least for this dataset, that the factors or the latent structures NMF and PCA-varimax are able to extract are superior representations of the discriminative information when compared to the variability, which basic PCA maximizes. Perhaps with a larger amount of data where more observations are available, PCA would achieve better performance.

There are several aspects that may influence the result we obtained in this paper such as the amount of data (observations), the number and the complexity of the contained materials, the noise levels, and the conditions in which reconstruction artifacts (e.g. beam hardening) are present. Nevertheless, the findings will shed a light for future investigations into utilizing the multispectral X-ray in an efficient way while considering those aspects.

\section{CONCLUSION}

We analyzed three linear methods we applied to reduce the dimensionality of the multispectral projection data prior to reconstruction. Using the reduced data where only a few, in our case 7, reconstructions are needed instead of 128 reconstructions needed otherwise, we were able to classify four different materials and achieve comparable results. This means that when the time-consuming iterative reconstruction methods are used, the overall computation time required to reconsult the multispectral data can be reduced significantly. Efficient reconstruction is crucial for utilizing the multispectral detectors in applications where rapid scanning is demanded. 

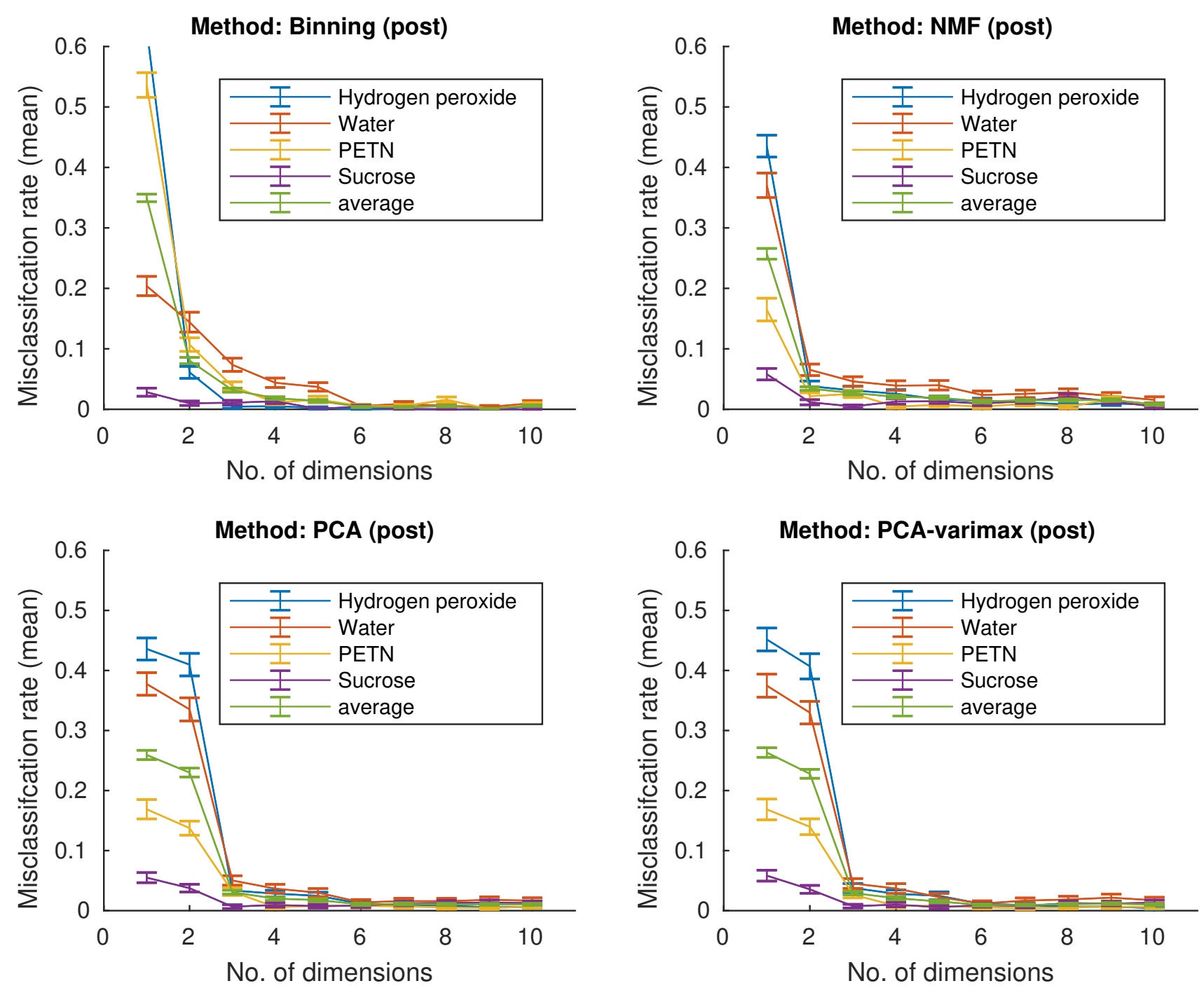

Figure 5: Classification performance when dimensionality reduction is performed on the reconstructed images (post-reconstruction reduction). Bars represent the standard deviations, however for better visibility, one fourth of the actual standard deviation is shown.

Future work will include studying this approach with data containing larger sets of material to assess its scalability. Moreover, we will investigate the impact of using linear methods to reduce the dimensionality of the projection data with respect to reconstruction artifacts such as beam hardening and metal artifacts.

\section{ACKNOWLEDGMENTS}

The Authors would like to acknowledge Innovation Fund Denmark for financing their respective work.

\section{REFERENCES}

[1] Maaß, C., Baer, M., and Kachelrieß, M., "Image-based dual energy CT using optimized precorrection functions: A practical new approach of material decomposition in image domain," Medical physics 36(8), 3818-3829 (2009). 


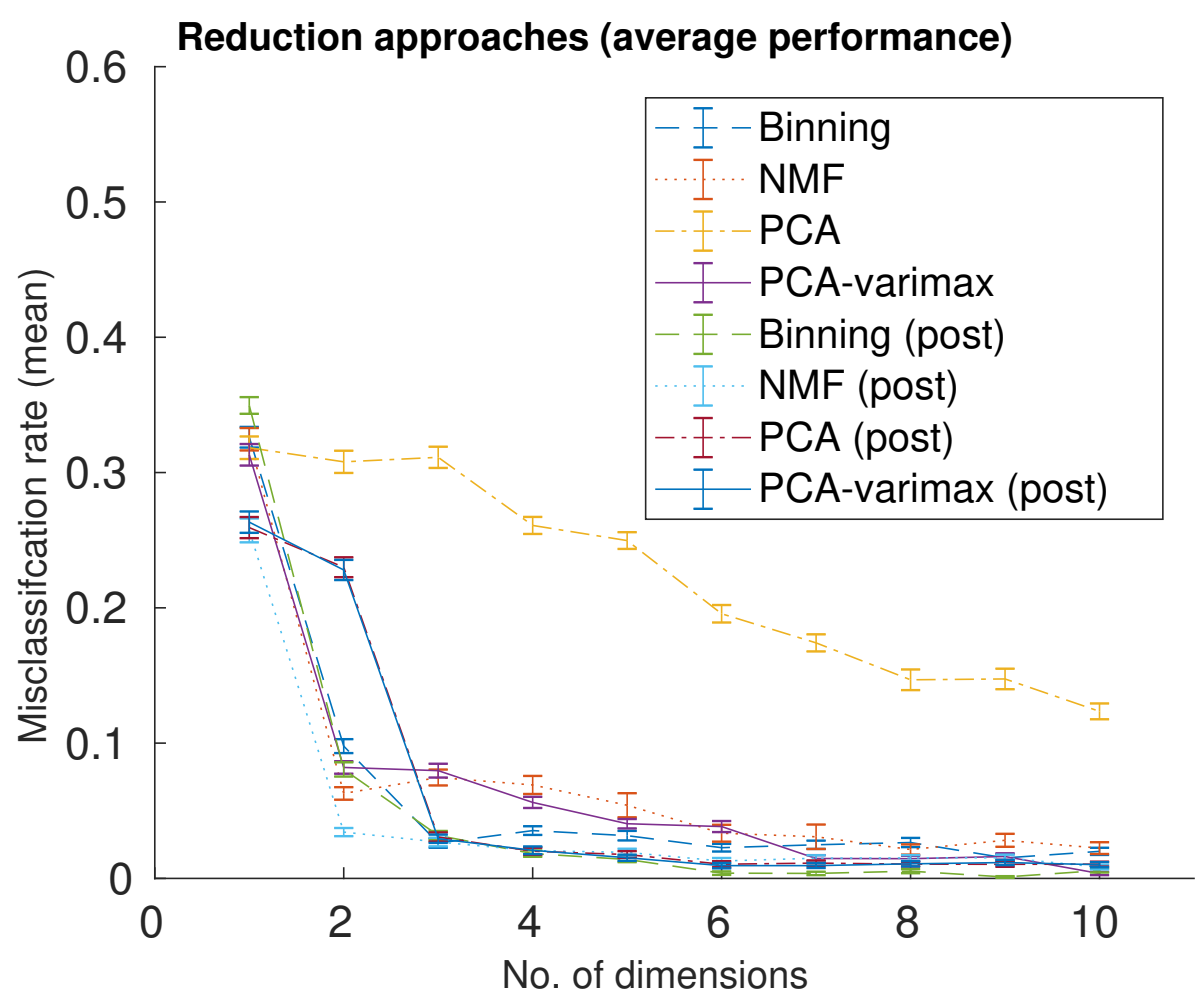

Figure 6: Average classification performance (summary of Figures 4 and 5). Bars represent the standard deviations, however for better visibility, one fourth of the actual standard deviation is shown.

[2] Einarsson, G., Jensen, J. N., Paulsen, R. R., Einarsdottir, H., Ersbøll, B. K., Dahl, A. B., and Christensen, L. B., "Foreign object detection in multispectral x-ray images of food items using sparse discriminant analysis," in [Scandinavian Conference on Image Analysis], 350-361, Springer (2017).

[3] Brambilla, A., Ouvrier-Buffet, P., Gonon, G., Rinkel, J., Moulin, V., Boudou, C., and Verger, L., "Fast CdTe and CdZnTe semiconductor detector arrays for spectroscopic x-ray imaging," IEEE Transactions on Nuclear Science 60(1), 408-415 (2013).

[4] Mouton, A. and Breckon, T. P., "A review of automated image understanding within 3D baggage computed tomography security screening," Journal of X-ray science and technology 23(5), 531-555 (2015).

[5] Kak, A. C. and Slaney, M., [Principles of computerized tomographic imaging], SIAM (2001).

[6] Rogers, T. W., Jaccard, N., Morton, E. J., and Griffin, L. D., "Automated x-ray image analysis for cargo security: Critical review and future promise," Journal of X-ray science and technology 25(1), 33-56 (2017).

[7] Kundegorski, M., Akçay, S., Devereux, M., Mouton, A., and Breckon, T., "On using feature descriptors as visual words for object detection within x-ray baggage security screening," in [International Conference on Imaging for Crime Detection and Prevention, IET],

[8] Akçay, S., Kundegorski, M. E., Devereux, M., and Breckon, T. P., "Transfer learning using convolutional neural networks for object classification within x-ray baggage security imagery," in [Image Processing (ICIP), 2016 IEEE International Conference on], 1057-1061, IEEE (2016).

[9] Johnson, T. R., Krauss, B., Sedlmair, M., Grasruck, M., Bruder, H., Morhard, D., Fink, C., Weckbach, S., Lenhard, M., Schmidt, B., et al., "Material differentiation by dual energy CT: initial experience," European radiology 17(6), 1510-1517 (2007).

[10] Graser, A., Johnson, T. R. C., Chandarana, H., and Macari, M., "Dual energy CT: preliminary observations and potential clinical applications in the abdomen," European Radiology 19(1), 13 (2008).

[11] Eger, L., Ishwar, P., Karl, W. C., and Pien, H., "Classification-aware dimensionality reduction methods for explosives detection using multi-energy x-ray computed tomography," in [Proc. SPIE, Computational Imaging IX], 7873, 78730Q-78730Q-7 (2011). 
[12] McCollough, C., Leng, S., Yu, L., and Fletcher, J., "Dual- and multi-energy CT: Principles, technical approaches, and clinical applications," Radiology 276, 637-653 (9 2015).

[13] Sidky, E. Y., Kao, C.-M., and Pan, X., "Accurate image reconstruction from few-views and limited-angle data in divergent-beam CT," Journal of X-ray Science and Technology 14(2), 119-139 (2006).

[14] Eger, L., Do, S., Ishwar, P., Karl, W. C., and Pien, H., "A learning-based approach to explosives detection using multi-energy x-ray computed tomography," in [2011 IEEE International Conference on Acoustics, Speech and Signal Processing (ICASSP)], 2004-2007 (May 2011).

[15] Lee, W.-J., Kim, D.-S., Kang, S.-R., Woo, S.-Y., and Yi, W.-J., "Material classification of multi-energy CT images using multiple discriminant analysis," in [Engineering in Medicine and Biology Society (EMBC), 2014 36th Annual International Conference of the IEEE], 1103-1106, IEEE (2014).

[16] Brambilla, A., Ouvrier-Buffet, P., Rinkel, J., Gonon, G., Boudou, C., and Verger, L., "CdTe linear pixel $\mathrm{x}$-ray detector with enhanced spectrometric performance for high flux x-ray imaging," IEEE Transactions on Nuclear Science 59(4), 1552-1558 (2012).

[17] Sidky, E. Y. and Pan, X., "Image reconstruction in circular cone-beam computed tomography by constrained, total-variation minimization," Physics in Medicine and Biology 53(17), 4777 (2008).

[18] Berry, M. W., Browne, M., Langville, A. N., Pauca, V. P., and Plemmons, R. J., "Algorithms and applications for approximate nonnegative matrix factorization," Computational statistics 83 data analysis $\mathbf{5 2}(1)$, $155-173$ (2007).

[19] Abdi, H. and Williams, L. J., "Principal component analysis," Wiley interdisciplinary reviews: computational statistics 2(4), 433-459 (2010).

[20] Kaiser, H. F., "The varimax criterion for analytic rotation in factor analysis," Psychometrika 23(3), 187-200 (1958).

[21] Kehres, J., Lyksborg, M., and Olsen, U. L., "Threat detection of liquid explosives and precursors from their x-ray scattering pattern using energy dispersive detector technology," in [Proc. SPIE, Radiation Detectors in Medicine, Industry, and National Security XVIII (accepted)], (2017).

[22] Christensen, E. D., Y, G., Kehres, J., Feidenhansl, R., and Olsen, U. L., "Spectral correction algorithm for multispectral CdTe x-ray detectors," in [Proc. SPIE, Radiation Detectors in Medicine, Industry, and National Security XVIII (accepted)], (2017). 